\title{
Stability Analysis of Complex-Valued Nonlinear Differential System
}

\author{
Tao Fang ${ }^{1,2}$ and Jitao Sun ${ }^{1}$ \\ ${ }^{1}$ Department of Mathematics, Tongji University, Shanghai 200092, China \\ ${ }^{2}$ School of Fundamental Studies, Shanghai University of Engineering Science, Shanghai 201620, China \\ Correspondence should be addressed to Jitao Sun; sunjt@sh163.net
}

Received 18 January 2013; Accepted 14 May 2013

Academic Editor: Kang Liu

Copyright (c) 2013 T. Fang and J. Sun. This is an open access article distributed under the Creative Commons Attribution License, which permits unrestricted use, distribution, and reproduction in any medium, provided the original work is properly cited.

\begin{abstract}
This paper studies the stability of complex-valued nonlinear differential system. The stability criteria of complex-valued nonlinear autonomous system are established. For the general complex-valued nonlinear non-autonomous system, the comparison principle in the context of complex fields is given. Those derived stability criteria not only provide a new method to analyze complex-valued differential system, but also greatly reduce the complexity of analysis and computation.
\end{abstract}

\section{Introduction}

The stability of differential system has been studied by many researchers, for example, [1-6] and references therein. However, the common setting adopted in aforementioned works is always in real number fields; namely, the objects of study are real-valued differential systems. The object of study in this paper is complex-valued differential system. Complex-valued differential system has also many potential applications in science and engineering. For example, quantum system and complex-valued neural networks are classical complex-valued differential system [7-10]. In fact, equations of many other classical systems, such as GinzburgLandau equation [11], Orr-Sommerfeld equation [12], complex Riccati equation [13], and complex Lorenz equation [14], are considered in the complex number fields. The usual method analyzing complex-valued system is to separate it into real part and imaginary part and then recast it into an equivalent real-valued system (see $[14,15]$ and references therein). But this method encounters two problems. One is that the dimension of the real-valued system is double that of complex-valued system, which leads to the difficulties on the analysis. Another is that this method needs an explicit separation of complex-valued function $f(t, z)$ into its real part and imaginary part; however, this separation is not always expressible in an analytical form. An efficient way to analyze complex-valued system is to retain the complex nature of system and consider its properties on $\mathbb{C}^{n}[16]$.

To the best of our knowledge, there have been few reports about the analysis and synthesis of complex-valued differential system except $[9,17-19]$, and there is no result so far about the stability of general complex-valued nonlinear differential system. In this paper, the stability criteria of complex-valued autonomous system are derived by the theory of several complex variable functions. Furthermore, the comparison principle of complex-valued nonautonomous differential system is given. Those derived stability criteria not only generalize some known results in literature but also greatly reduce the complexity of analysis and computation. As an application, the stability conditions of a class of complexvalued nonlinear systems are presented. Those problems are meaningful and challenging.

The remainder of the paper is organized as follows. In Section 2, the complex-valued system to be dealt with is formulated and several results about the complex-valued functions are presented. The stability criteria of complexvalued autonomous system are established in Section 3. Moreover, the comparison principle of complex-valued nonlinear system is given in Section 4. Based on the comparison principle, the stability conditions of a class of complex-valued 
systems are also presented in Section 4. The main points of the paper are illustrated by some examples in the corresponding sections. Finally, some conclusions are drawn in Section 5.

\section{Notations and Preliminaries}

In this section, we introduce notations, definitions, and preliminary facts which are used throughout this paper.

Let $\Omega \subseteq \mathbb{C}^{n}$ be a neighbourhood of the origin, $I=$ $\left[t_{0},+\infty\right), t_{0} \geq 0 . W(z) \in C[\Omega, \mathbb{R}], W(0)=0, V(t, z) \in C[I \times$ $\Omega, \mathbb{R}], V(t, 0)=0 . H\left[\Omega, \mathbb{C}^{n}\right]$ denote all holomorphic maps from $\Omega$ to $\mathbb{C}^{n}$. Let $\bar{z}$ and $z^{*}$ be the conjugate and conjugate transpose of $z=\left(z_{1}, z_{2}, \ldots, z_{n}\right)^{T} \in \mathbb{C}^{n}$, respectively, and $\|z\|=\sqrt{z^{*} z}, i=\sqrt{-1}$.

Definition 1. $W(z)$ is called a complex positive definite function on $\Omega$, if $W(z) \geq 0$ for any $z \in \Omega$, and $W(z)=0$ if and only if $z=0$.

Definition 2. $V(t, z)$ is called a positive definite function on $I \times \Omega$ if there exists a complex positive definite function $W(z)$ such that $V(t, z) \geq W(z)$ for any $z \in \Omega$, and $V(t, z)=0$ if and only if $z=0$.

In this paper, we will study the stability of complex-valued nonlinear differential system described by

$$
\begin{aligned}
& \frac{d z}{d t}=f(t, z(t)), \\
& z\left(t_{0}\right)=z_{0} \in \mathbb{C}^{n},
\end{aligned}
$$

where $t_{0} \in R^{+}, f: I \times \Omega \mapsto \mathbb{C}^{n}$ is a complexvalued continuous map. $z: I \mapsto \mathbb{C}^{n}$ is a complex-valued differentiable map. Let $z_{e}$ be an equilibrium point satisfying $f\left(t, z_{e}\right)=0 ; z(t)=z\left(t, t_{0}, z_{0}\right)$ satisfies system $(1)$.

Definition 3. System (1) is said to be stable at $z_{e}$, if for all $\varepsilon>0$, $\exists \delta\left(\varepsilon, t_{0}\right)$ such that $\left\|z_{0}-z_{e}\right\|<\delta$ implies

$$
\left\|z(t)-z_{e}\right\|<\varepsilon, \quad \forall t \geq t_{0} .
$$

Definition 4. System (1) is said to be asymptotically stable at $z_{e}$ if it is stable and $\lim _{t \rightarrow \infty}\left\|z(t)-z_{e}\right\|=0$.

Lemma 5 (see [20]). Let $g \in C\left[R^{+} \times R^{+}, R\right], m \in C\left[R^{+}, R^{+}\right]$, and $\operatorname{Dm}(t) \leq g(t, m(t))$, for all $t \in\left[t_{0},+\infty\right) \backslash S$, where $S$ is a countable subset of $\left[t_{0}, \infty\right)$ and $D$ is any one of the Dini derivatives. Suppose that the maximal solution $r(t)$ of

$$
\dot{u}(t)=g(t, u(t)), \quad u\left(t_{0}\right)=u_{0} \geq 0, \quad t_{0} \in R^{+}
$$

exists on $\left[t_{0}, \infty\right)$ and $m\left(t_{0}\right) \leq u_{0}$. Then $m(t) \leq r(t), t \geq t_{0}$.

Lemma 6. For any positive definite continuous function $W(z)$ on $\Omega=\left\{z \in \mathbb{C}^{n} \mid\|z\| \leq H\right\}, H>0$, there exist two $K$ class functions $a(\cdot)$ and $b(\cdot)$ such that

$$
a(\|z\|) \leq W(z) \leq b(\|z\|) .
$$

The proof of Lemma 6 is similar to the case of positive definition functions defined in the real fields [21].

\section{Stability Criteria of Complex-Valued Autonomous System}

In this section, we will study the stability of complex-valued autonomous system described by

$$
\begin{gathered}
\frac{d z}{d t}=f(z), \\
z\left(t_{0}\right)=z_{0} \in \mathbb{C}^{n},
\end{gathered}
$$

where $f(z)=\left(f_{1}(z), \ldots, f_{n}(z)\right)^{T}, z_{\alpha} \in \mathbb{C}, f_{\alpha}(z): \Omega \mapsto \mathbb{C}$, $f\left(z_{e}\right)=0, \alpha=1, \ldots, n$.

By the substitutions of $z_{\alpha}=x_{\alpha}+i y_{\alpha}$ and $f_{\alpha}(z)=$ $u_{\alpha}(x, y)+i v_{\alpha}(x, y)$, system (5) can be recast into the following real-valued system:

$$
\begin{aligned}
& \dot{x}=u(x, y), \\
& \dot{y}=v(x, y),
\end{aligned}
$$

where $x=\left(x_{1}, \ldots, x_{n}\right)^{T}, y=\left(y_{1}, \ldots, y_{n}\right)^{T}, u=\left(u_{1}, \ldots, u_{n}\right)^{T}$, and $v=\left(v_{1}, \ldots, v_{n}\right)^{T}$. The homomorphism between the complex-valued system (5) and the real-valued system (6) allows us to employ the existing methods to analyze the stability of the complex system (5). We linearize system (6):

$$
\left(\begin{array}{c}
\dot{x} \\
\dot{y}
\end{array}\right)=J\left(x_{e}, y_{e}\right)\left(\begin{array}{l}
x \\
y
\end{array}\right)
$$

where

$$
J\left(x_{e}, y_{e}\right)=\left[\frac{\partial\left(u_{1}, \ldots, u_{n}, v_{1}, \ldots, v_{n}\right)}{\partial\left(x_{1}, \ldots, x_{n}, y_{1}, \ldots, y_{n}\right)}\right]_{\left(x_{e}, y_{e}\right)}
$$

is the Jacobi matrix of $\left(u_{1}, \ldots, u_{n}, v_{1}, \ldots, v_{n}\right)$ evaluated at $z_{e}=$ $x_{e}+i y_{e}$. Therefore, judging the stability of a nonlinear system is reduced to the problem of finding the eigenvalues of its related Jacobi matrix. The evaluation of $J\left(x_{e}, y_{e}\right)$ needs an explicit separation of the complex functions $f_{\alpha}(z)$ into its real part and imaginary part. This separation is not always expressible in an analytical form. We can evaluate $J\left(x_{e}, y_{e}\right)$ by manipulating $f(z)$ directly without resorting to its real and imaginary parts. Next we will discuss two cases.

Case 1. $f(z)$ is holomorphic map, namely, $f(z) \in H\left[\Omega, \mathbb{C}^{n}\right]$. In this case, since $f_{\alpha}(z)$ is analytic function, $f_{\alpha}(z)$ satisfies Cauchy-Riemann equation, and the following equalities hold for $\alpha, j=1,2, \ldots, n$ :

$$
\begin{gathered}
\frac{\partial u_{\alpha}}{\partial x_{j}}=\frac{\partial v_{\alpha}}{\partial y_{j}}, \quad \frac{\partial u_{\alpha}}{\partial y_{j}}=-\frac{\partial v_{\alpha}}{\partial x_{j}} \\
\frac{\partial f_{\alpha}}{\partial z_{j}}=\frac{\partial u_{\alpha}}{\partial x_{j}}+i \frac{\partial v_{\alpha}}{\partial x_{j}} .
\end{gathered}
$$


Denote

$$
\begin{gathered}
\frac{\partial u}{\partial x} \triangleq \frac{\partial\left(u_{1}, \ldots, u_{n}\right)}{\partial\left(x_{1}, \ldots, x_{n}\right)}, \quad \frac{\partial u}{\partial y} \triangleq \frac{\partial\left(u_{1}, \ldots, u_{n}\right)}{\partial\left(y_{1}, \ldots, y_{n}\right)}, \\
\frac{\partial v}{\partial x} \triangleq \frac{\partial\left(v_{1}, \ldots, v_{n}\right)}{\partial\left(x_{1}, \ldots, x_{n}\right)}, \quad \frac{\partial v}{\partial y} \triangleq \frac{\partial\left(v_{1}, \ldots, v_{n}\right)}{\partial\left(y_{1}, \ldots, y_{n}\right)}, \\
\frac{\partial f}{\partial z} \triangleq \frac{\partial\left(f_{1}, \ldots, f_{n}\right)}{\partial\left(z_{1}, \ldots, z_{n}\right)} .
\end{gathered}
$$

It follows from (9) that

$$
\begin{gathered}
\frac{\partial u}{\partial x}=\frac{\partial v}{\partial y}, \quad \frac{\partial u}{\partial y}=-\frac{\partial v}{\partial x} \\
\frac{\partial f}{\partial z}=\frac{\partial u}{\partial x}+i \frac{\partial v}{\partial x} .
\end{gathered}
$$

Thus

$$
\begin{aligned}
& \frac{1}{2}\left[\begin{array}{cc}
I_{n} & -i I_{n} \\
I_{n} & i I_{n}
\end{array}\right] J\left(x_{e}, y_{e}\right)\left[\begin{array}{cc}
I_{n} & I_{n} \\
i I_{n} & -i I_{n}
\end{array}\right] \\
& =\frac{1}{2}\left[\begin{array}{cc}
\frac{\partial u}{\partial x}-i \frac{\partial u}{\partial y} & \frac{\partial v}{\partial x}-i \frac{\partial v}{\partial y} \\
\frac{\partial u}{\partial x}+i \frac{\partial u}{\partial y} & \frac{\partial v}{\partial x}+i \frac{\partial v}{\partial y}
\end{array}\right]\left[\begin{array}{cc}
I_{n} & I_{n} \\
i I_{n} & -i I_{n}
\end{array}\right] \\
& =\frac{1}{2}\left[\begin{array}{cc}
\frac{\partial u}{\partial x}-i \frac{\partial u}{\partial y}+i \frac{\partial v}{\partial x}+\frac{\partial v}{\partial y} & \frac{\partial u}{\partial x}-i \frac{\partial u}{\partial y}-i \frac{\partial v}{\partial x}-\frac{\partial v}{\partial y} \\
\frac{\partial u}{\partial y}+i \frac{\partial v}{\partial x}-\frac{\partial v}{\partial y} & \frac{\partial u}{\partial x}+i \frac{\partial u}{\partial y}-i \frac{\partial v}{\partial x}+\frac{\partial v}{\partial y}
\end{array}\right] \\
& =\left[\begin{array}{c}
\frac{\partial u}{\partial x}+i \frac{\partial v}{\partial x} \quad 0 \quad \frac{\partial u}{\partial x}-i \frac{\partial v}{\partial x} \\
0
\end{array}\right]=C J\left(z_{e}\right)
\end{aligned}
$$

where $J\left(x_{e}, y_{e}\right)$ can be rewritten by

$$
\begin{gathered}
J\left(x_{e}, y_{e}\right)=\left[\begin{array}{ll}
\frac{\partial u}{\partial x} & \frac{\partial v}{\partial x} \\
\frac{\partial u}{\partial y} & \frac{\partial v}{\partial y}
\end{array}\right]_{\left(x_{e}, y_{e}\right)} \\
C J\left(z_{e}\right)=\left[\begin{array}{cc}
\frac{\partial f}{\partial z} & 0 \\
0 & \left.\frac{\partial f}{\partial z}\right)
\end{array}\right]_{z_{e}}
\end{gathered}
$$

The equality (12) means that $J\left(x_{e}, y_{e}\right)$ and $C J\left(z_{e}\right)$ are similar matrices; thus they have the same eigenvalues. Moreover, we notice that if $\lambda$ is an eigenvalue of $\partial f / \partial z$, then $\bar{\lambda}$ is an eigenvalue of $\overline{\partial f / \partial z}$. It turns out that the eigenvalues of $J\left(x_{e}, y_{e}\right)$ are merely the eigenvalues of $[\partial f / \partial z]_{z_{e}}$ if we do not consider the multiplicity of the eigenvalues. Therefore, the stability of (7) can be easily checked by examining whether the real part of the eigenvalues of

$$
J\left(z_{e}\right)=\left[\frac{\partial\left(f_{1}, \ldots, f_{n}\right)}{\partial\left(z_{1}, \ldots, z_{n}\right)}\right]_{z_{e}}
$$

is negative. We conclude the following results.

Theorem 7. For system (5) such that $f(z)$ is holomorphic,

(i) if all of the eigenvalues of $J\left(z_{e}\right)$ lie on the left half complex plane, system (5) is exponentially asymptotically stable at $z_{e}$;

(ii) if there exists an eigenvalue of $J\left(z_{e}\right)$ lying on the right half complex plane, system (5) is not stable at $z_{e}$;

(iii) if there is no eigenvalue of $J\left(z_{e}\right)$ lying on the right half complex plane, and there are a pair of eigenvalues lying on the imaginary axis at least, the stability of system (5) at $z_{e}$ is not clear.

Corollary 8. For system (5) such that $n=1$ and $f(z)$ is holomorphic,

(i) if the real part of $f^{\prime}\left(z_{e}\right)<0$, system (5) is exponentially asymptotically stable at $z_{e}$;

(ii) if the real part of $f^{\prime}\left(z_{e}\right)>0$, system (5) is not stable at $z_{e}$;

(iii) if the real part of $f^{\prime}\left(z_{e}\right)=0$, the stability of system (5) at $z_{e}$ is not clear.

Case 2. $f(z)$ is not holomorphic. In particular, we consider the case that $f(z)=g(z, \bar{z})$, where $g(\cdot, \cdot) \in H\left[\Omega \times \Omega, \mathbb{C}^{2 n}\right]$.

The motivation discussing this case is that some wellknown complex-valued systems, such as complex Lorenz system [14], complex Chen system [15] and complex Lü system [15], belong to this case. For the details see Example 13. In this case, since $f(z)$ is not holomorphic in $z, f_{\alpha}(z)$ does not satisfy Cauchy-Riemann equation again. But we have the following equalities:

$$
\begin{gathered}
u_{\alpha}(x, y)=\frac{1}{2}\left[g_{\alpha}(z, \bar{z})+\bar{g}_{\alpha}(z, \bar{z})\right], \\
v_{\alpha}(x, y)=-\frac{i}{2}\left[g_{\alpha}(z, \bar{z})-\bar{g}_{\alpha}(z, \bar{z})\right], \\
\frac{\partial u_{\alpha}}{\partial x_{j}}=\frac{1}{2}\left[\frac{\partial g_{\alpha}}{\partial z_{j}}+\frac{\partial g_{\alpha}}{\partial \bar{z}_{j}}+\frac{\partial \bar{g}_{\alpha}}{\partial z_{j}}+\frac{\partial \bar{g}_{\alpha}}{\partial \bar{z}_{j}}\right], \\
\frac{\partial u_{\alpha}}{\partial y_{j}}=\frac{i}{2}\left[\frac{\partial g_{\alpha}}{\partial z_{j}}-\frac{\partial g_{\alpha}}{\partial \bar{z}_{j}}+\frac{\partial \bar{g}_{\alpha}}{\partial z_{j}}-\frac{\partial \bar{g}_{\alpha}}{\partial \bar{z}_{j}}\right], \\
\frac{\partial v_{\alpha}}{\partial x_{j}}=-\frac{i}{2}\left[\frac{\partial g_{\alpha}}{\partial z_{j}}+\frac{\partial g_{\alpha}}{\partial \bar{z}_{j}}-\frac{\partial \bar{g}_{\alpha}}{\partial z_{j}}-\frac{\partial \bar{g}_{\alpha}}{\partial \bar{z}_{j}}\right], \\
\frac{\partial v_{\alpha}}{\partial y_{j}}=\frac{1}{2}\left[\frac{\partial g_{\alpha}}{\partial z_{j}}-\frac{\partial g_{\alpha}}{\partial \bar{z}_{j}}-\frac{\partial \bar{g}_{\alpha}}{\partial z_{j}}+\frac{\partial \bar{g}_{\alpha}}{\partial \bar{z}_{j}}\right] .
\end{gathered}
$$


From (15), we have $P^{-1} \widetilde{J}\left(x_{e}, y_{e}\right) P=\widetilde{J}\left(z_{e}\right)$, where

$$
\begin{gathered}
P^{-1}=\frac{1}{2}\left[\begin{array}{cc}
(i-1) I_{n} & i I_{n} \\
(i-1) I_{n} & I_{n}
\end{array}\right], \\
P=\left[\begin{array}{cc}
-i I_{n} & -I_{n} \\
-(1+i) I_{n} & (1+i) I_{n}
\end{array}\right], \\
\widetilde{J}\left(x_{e}, y_{e}\right)=\left[\begin{array}{ll}
\frac{\partial\left(u_{1}, \ldots, u_{n}\right)}{\partial\left(x_{1}, \ldots, x_{n}\right)} & \frac{\partial\left(u_{1}, \ldots, u_{n}\right)}{\partial\left(x_{1}, \ldots, x_{n}\right)} \\
\frac{\partial\left(v_{1}, \ldots, v_{n}\right)}{\partial\left(y_{1}, \ldots, y_{n}\right)} & \frac{\partial\left(v_{1}, \ldots, v_{n}\right)}{\partial\left(y_{1}, \ldots, y_{n}\right)}
\end{array}\right]_{\left(x_{e}, y_{e}\right)}, \\
\widetilde{J}\left(z_{e}\right)=\left[\begin{array}{ll}
\frac{\partial\left(g_{1}, \ldots, g_{n}\right)}{\partial\left(z_{1}, \ldots, z_{n}\right)} & -i \frac{\partial\left(g_{1}, \ldots, g_{n}\right)}{\partial\left(\bar{z}_{1}, \ldots, \bar{z}_{n}\right)} \\
i \frac{\partial\left(\bar{g}_{1}, \ldots, \bar{g}_{n}\right)}{\partial\left(z_{1}, \ldots, z_{n}\right)} & \frac{\partial\left(\bar{g}_{1}, \ldots, \bar{g}_{n}\right)}{\partial\left(\bar{z}_{1}, \ldots, \bar{z}_{n}\right)}
\end{array}\right]_{z_{e}} .
\end{gathered}
$$

Hence, $\widetilde{J}\left(x_{e}, y_{e}\right)$ and $\widetilde{J}\left(z_{e}\right)$ are similar matrices; they have the same eigenvalues. We conclude the following:

Theorem 9. For system (5) with $f(z)=g(z, \bar{z})$,

(i) if all of eigenvalues of $\widetilde{J}\left(z_{e}\right)$ lie on the left half complex plane, then system (5) is exponentially asymptotically stable at $z_{e}$;

(ii) if there exists an eigenvalue of $\widetilde{J}\left(z_{e}\right)$ lying on the right half complex plane, then system (5) is not stable at $z_{e}$;

(iii) if there is no eigenvalue of $\widetilde{J}\left(z_{e}\right)$ lying on the right half complex plane, and there are a pair of eigenvalues lying on the imaginary axis at least, then the stability of system (5) at $z_{e}$ is not clear.

Remark 10. Theorems 7 and 9 provide a new method to analyze complex-valued differential system.

Remark 11. Corollary 8 is the existing results in [9].

Example 12. Consider the stability of the trivial solution of a class complex-valued Riccati equation defined by

$$
\frac{d z}{d t}=z^{n}-p_{n-1} z^{n-1}+\cdots+p_{1} z
$$

where $n$ is a positive integer, $p_{j} \in \mathbb{C}, j=1, \ldots, n$.

In this example, $f(z)=z^{n}-p_{n-1} z^{n-1}+\cdots+p_{1} z, f^{\prime}(0)=$ $p_{1}$; by Corollary 8 , if the real part of $p_{1}$ is negative, system (17) is exponentially asymptotically stable at $z_{e}=0$; if the real part of $p_{1}$ is positive, system (17) is not stable at $z_{e}=0$.

Example 13. Consider the following system:

$$
\begin{gathered}
\dot{z}_{1}=z_{1}^{2}-i z_{1}+z_{2}, \\
\dot{z}_{2}=-2 z_{1}-z_{2}, \\
z(0)=z_{0} \in \mathbb{C} .
\end{gathered}
$$

System (18) has two equilibrium points $P_{1}(0,0)$ and $P_{2}(2+$ $i,-4-2 i)$, and

$$
J\left(P_{1}\right)=\left(\begin{array}{cc}
-i & 1 \\
-2 & -1
\end{array}\right), \quad J\left(P_{2}\right)=\left(\begin{array}{cc}
4+i & 1 \\
-2 & -1
\end{array}\right)
$$

The eigenvalues of $J(0,0)$ and $J(2+i,-4-2 i)$ are $\lambda_{1}=$ $-432 / 1331-899 / 467 i, \lambda_{2}=-899 / 1331+432 / 467 i$ and $\lambda_{3}=1575 / 439+1313 / 1195 i, \lambda_{4}=-841 / 1431-118 / 1195 i$, respectively. By Theorem 7 , system (18) is exponentially asymptotically stable at $P_{1}$ and not stable at $P_{2}$.

An alternative, but tedious, approach to the problem is to separate (18) into real and imaginary parts and rewrite it as (6):

$$
\begin{gathered}
\dot{x}_{1}=x_{1}^{2}+x_{2}+y_{1}-y_{1}^{2}, \\
\dot{x}_{2}=-2 x_{1}-x_{2}, \\
\dot{y}_{1}=2 x_{1} y_{1}-x_{1}+y_{2}, \\
\dot{y}_{2}=-2 y_{1}-y_{2} .
\end{gathered}
$$

The equilibrium points of system $(20)$ are $P_{1}(0,0,0,0)$ and $P_{2}(2,-4,1,-2)$. To judge the stability of those equilibrium points, we have to compute the eigenvalue of Jacobi matrix:

$$
J(x, y)=\left(\begin{array}{cccc}
2 x_{1} & 1 & 1-2 y_{1} & 0 \\
-2 & -1 & 0 & 0 \\
2 y_{1}-1 & 0 & 2 x_{1} & 1 \\
0 & 0 & -2 & -1
\end{array}\right) \text {. }
$$

By calculating, it can be found that $P_{1}$ are stable and $P_{2}$ are not stable; it is not contradictory; in fact, $P_{1}$ is just the point $(0,0)$ and $P_{2}$ is just $(2+i,-4-2 i)$ on $\mathbb{C}^{2}$.

Remark 14 . The common method to analyze the complexvalued systems is very tedious and complex. Theorem 7 not only generalizes some known results in the literature [9], but also greatly reduces the complexity of analysis and computation.

Example 15. In 2007, Mahmoud presented complex-valued Chen system [15] described by

$$
\begin{gathered}
\dot{z}_{1}=\alpha\left(z_{2}-z_{1}\right), \\
\dot{z}_{2}=(\gamma-\alpha) z_{1}-z_{1} z_{3}+\gamma z_{2}, \\
\dot{z}_{3}=\frac{1}{2}\left(\bar{z}_{1} z_{2}+z_{1} \bar{z}_{2}\right)-\beta z_{3},
\end{gathered}
$$

where $\alpha, \beta$, and $\gamma$ are positive real parameters; $z_{1}, z_{2} \in \mathbb{C}$ are complex state variables; and $z_{3} \in \mathbb{R}$ is real state variables. Obviously, $f(z)=\left(\alpha\left(z_{2}-z_{1}\right),(\gamma-\alpha) z_{1}-z_{1} z_{3}+\gamma z_{2},\left(\bar{z}_{1} z_{2}+\right.\right.$ $\left.\left.z_{1} \bar{z}_{2}\right) / 2-\beta z_{3}\right)$ is not holomorphic because of the existence of 
$\bar{z}_{1}$ and $\bar{z}_{2}$. For simplicity, we discuss only the stability of the origin. According to (16), we have

$$
\widetilde{J}(0)=\left[\begin{array}{cc}
A & 0 \\
0 & A
\end{array}\right], \quad \text { where } A=\left[\begin{array}{ccc}
-\alpha & \alpha & 0 \\
\gamma-\alpha & \gamma & 0 \\
0 & 0 & -\beta
\end{array}\right] .
$$

By Theorem 9, it is easy to conclude that the origin of system (22) is exponentially asymptotically stable when $\alpha>2 \gamma$.

Remark 16. It is not necessary to analyze the stability of system (22) by decoupling it into real and imaginary parts just like [15]. The conclusion in Example 15 is just the existing result in [15]. Similarly, the stability of complex-valued Lorenz system and Lü system can be derived by Theorem 9 .

\section{Stability of Complex-Valued Nonautonomous System}

In this section, the comparison principle of system (1) is presented.

Theorem 17. Assume that there exists a positive definite function $V(t, z) \in C\left[I \times \Omega, \mathbb{R}^{+}\right]$such that $V(t, z)$ is locally Lipschitzian in $z$, and

$$
\left.D^{+} V(t, z(t))\right|_{(1)} \leq g(t, V(t, z(t))), \quad t \in I \backslash S,
$$

where $I=\left[t_{0},+\infty\right), S$ is a countable subset of $I, g \in C[I \times$ $\left.R^{+}, R\right]$, and $\Omega \subseteq \mathbb{C}^{n}$ is a neighbourhood of the origin. Then the stability properties of the trivial solution of (3) with $g(t, 0)=$ 0 imply the corresponding stability properties of the trivial solution of $(1)$ such that $f(t, 0)=0$.

Proof. We just prove the case that the trivial solution of system (3) is stable.

Since $V(t, z)$ is a positive definite function, by Lemma 6 , there exists a $K$ class function $a(\cdot)$ such that

$$
V(t, z) \geq a(\|z\|) .
$$

Considering the stability of the trivial solution of (3), for all $\varepsilon>0, \exists \delta^{*}\left(\varepsilon, t_{0}\right)$, when $0<u_{0}<\delta^{*}$, the solution of (3) satisfies

$$
u\left(t, t_{0}, u_{0}\right)<a(\varepsilon) .
$$

Considering the continuity of $V(t, z)$ and the fact $V(t, 0)=0$, $\exists \delta\left(\varepsilon, t_{0}\right)$, when $\left\|z_{0}\right\|<\delta$, we have $0<V\left(t_{0}, z_{0}\right) \triangleq V_{0}<u_{0}<$ $\delta^{*}$. Let $z(t)=z\left(t, t_{0}, z_{0}\right)$ be the solution of system (1), then

$$
\begin{gathered}
\left.D^{+} V\right|_{(1)} \leq g(t, V), \quad t \in I \backslash S, \\
V\left(t_{0}, z_{0}\right)=V_{0} .
\end{gathered}
$$

By Lemma 5, we have

$$
a(\|z(t)\|) \leq V(t, z(t)) \leq u\left(t, t_{0}, u_{0}\right)<a(\varepsilon),
$$

so $\|z(t)\|<\varepsilon$; thus the trivial solution of (1) is stable. The proof is completed.
Example 18. Consider the stability of the trivial solution of the following system:

$$
\begin{gathered}
\dot{z}_{1}=(-3+8 \sin t) z_{1}+(\sin t) z_{2}, \\
\dot{z}_{2}=(\cos t) z_{1}+(-3+8 \sin t) z_{2}, \\
z(0)=z_{0} \in \mathbb{C}^{2} .
\end{gathered}
$$

Let $V(t, z)=(1 / 2)\left(\left\|z_{1}\right\|^{2}+\left\|z_{2}\right\|^{2}\right)$, then

$$
\begin{aligned}
\left.\frac{d V}{d t}\right|_{(29)}= & \frac{1}{2}\left(\dot{z}_{1} \bar{z}_{1}+z_{1} \dot{\bar{z}}_{1}+\dot{z}_{2} \bar{z}_{2}+z_{2} \dot{\bar{z}}_{2}\right) \\
= & (-3+8 \sin t)\left(\left\|z_{1}\right\|^{2}+\left\|z_{2}\right\|^{2}\right) \\
& +(\sin t+\cos t) \operatorname{Re}\left(\bar{z}_{1} z_{2}\right) \\
\leq & (-3+8 \sin t)\left(\left\|z_{1}\right\|^{2}+\left\|z_{2}\right\|^{2}\right) \\
& +2\left\|z_{1} z_{2}\right\| \\
\leq & (-3+8 \sin t)\left(\left\|z_{1}\right\|^{2}+\left\|z_{2}\right\|^{2}\right) \\
& +\left\|z_{1}\right\|^{2}+\left\|z_{2}\right\|^{2} \\
= & (-2+8 \sin t)\left(\left\|z_{1}\right\|^{2}+\left\|z_{2}\right\|^{2}\right) \\
= & 2(-2+8 \sin t) V \triangleq g(t, V) .
\end{aligned}
$$

Since the solution of system

$$
\begin{gathered}
\dot{u}=g(t, u)=2(-2+8 \sin t) u, \\
u(0)=u_{0} \in \mathbb{R}
\end{gathered}
$$

is $u(t)=u_{0} \exp \{-16-4 t-16 \cos t\}$, the trivial solution of system (29) is also asymptotically stable by Theorem 17 .

Remark 19. Just as Example 13, we can separate (29) into real and imaginary parts and rewrite it as a 4-dimension real time-varying system and then use the comparison principle in the context of real fields to judge the stability of (29), but the computation is more complex and lengthy. In fact, Theorem 17 is the generalization of the real comparison principle in the complex fields, we can judge directly the stability properties of complex-valued differential system by Theorem 17.

Generally speaking, the construction of the complex positive definite function $V(t, z)$ is more difficult than the case of real system. Next we will give the constructing method of complex Lyapunov function of a class complex-valued system defined by

$$
\dot{z}=G(t, z)[h(z)+g(t, z)],
$$

where $G \in C\left[I \times \Omega, \mathbb{R}^{+}\right], g \in C[I \times \Omega, \mathbb{C}], I=\left[t_{0},+\infty\right)$, $t_{0} \geq 0, \Omega \subseteq \mathbb{C}$ is a neighbourhood of the origin, $h \in H[\Omega, \mathbb{C}]$, $h^{\prime}(0) \neq 0$, and $h(0)=0$ if and only if $z=0$. 
System (32) has been discussed to study the asymptotic stability of complex-valued Riccati equation

$$
\dot{z}=q(t, z)-p(t) z^{2}
$$

in [18]. Next we will give the exponentially asymptotic stability condition of system (32) by Theorem 17. Let

$$
r(z)= \begin{cases}\frac{z h^{\prime}(0)-h(z)}{z h(z)}, & z \in \Omega, z \neq 0, \\ -\frac{h^{\prime \prime}(0)}{2 h^{\prime}(0)}, & z=0 .\end{cases}
$$

It can be verified that $r(z)$ is analytic function in $\Omega$, so we can take

$$
w(z)=z \exp \left\{\int_{0}^{z} r(t) d t\right\}
$$

and $V(z)=\|w(z)\|$, then when $h(z) \neq 0$,

$$
\begin{aligned}
\left.\frac{d}{d t} V^{2}(z)\right|_{(32)} & =\frac{d}{d t}[w(z) \overline{w(z)}] \\
& =2 \operatorname{Re}[\dot{w}(z) \overline{w(z)} \dot{z}] \\
& =2 \operatorname{Re}\left\{w(z) \overline{w(z)}\left[z^{-1}+r(z) \dot{z}\right]\right\} \\
& =2 V^{2}(z) \operatorname{Re}\left[h^{\prime}(0) h^{-1}(z) \dot{z}\right],
\end{aligned}
$$

thus

$$
\begin{aligned}
\left.\frac{d}{d t} V(z)\right|_{(32)}= & V(z) \operatorname{Re}\left[h^{\prime}(0) h^{-1}(z) \dot{z}\right] \\
= & G(t, z) V(z) \\
& \times \operatorname{Re}\left\{h^{\prime}(0) h^{-1}(z)[h(z)+g(t, z)]\right\} \\
= & G(t, z) V(z) \operatorname{Re}\left\{h^{\prime}(0)\left[1+\frac{g(t, z)}{h(z)}\right]\right\} .
\end{aligned}
$$

By Theorem 17, when $\left.\dot{V}(z)\right|_{(32)} \leq-c V(z), c>0$, namely,

$$
G(t, z) \operatorname{Re}\left\{h^{\prime}(0)\left[1+\frac{g(t, z)}{h(z)}\right]\right\} \leq-c,
$$

the trivial solution of system (32) is exponentially asymptotically stable. So we have the following result.

Theorem 20. The trivial solution of system (32) is exponentially asymptotically stable if $\exists c>0$ such that the inequality (38) holds for almost everywhere $(t, z) \in I \times \Omega$.

Example 21. Consider complex-valued Riccati equation defined by

$$
\begin{gathered}
\frac{d z}{d t}=-e^{-t}\left[(\rho+t) z+z^{2}\right], \\
z(0)=z_{0} \in \mathbb{C},
\end{gathered}
$$

where $t \geq 0$ and $\|z\|<\rho, \rho>0$. In this example, $G(t, z)=e^{-t}$, $h(z)=-z^{2}-\rho z, g(t, z)=-z t$, and when $h(z) \neq 0$

$$
\begin{aligned}
& G(t, z) \operatorname{Re}\left\{h^{\prime}(0)\left[1+\frac{g(t, z)}{h(z)}\right]\right\} \\
& \quad<-\rho \operatorname{Re}\left\{1+\frac{t}{\rho+z}\right\}=-\rho\left\{1+\frac{\rho+\operatorname{Re} z}{\|\rho+z\|^{2}} t\right\}<-\rho .
\end{aligned}
$$

So the trivial solution of (39) is exponentially asymptotically stable.

Remark 22. The asymptotic stability of system (39) can not be judged by Theorem 3.2 of the paper [18], because condition (3.13) of the theorem can not be satisfied, so our stability criterion is more effective.

\section{Conclusion}

This paper has discussed the stability of complex-valued differential system. The stability criteria of complex-valued autonomous system has been established. For the general complex-valued non-autonomous system, the comparison principle in the context of complex fields have been derived. Those derived stability criteria not only provide a new method to analyze complex-valued differential system, but also greatly reduce the complexity of analysis and computation. Based on the new results, the stability conditions of a class complex-valued nonlinear system have been presented.

\section{Acknowledgments}

The authors would like to thank the editor and the reviewers for their constructive comments and suggestions which improved the quality of the paper. This work is supported by the NNSF of China under Grants 61174039 and 61203128 and the Connotative Construction Project of Shanghai University of Engineering Science under Grants nhky-2012-13.

\section{References}

[1] H. Yan, S. Ma, Y. Liu, and H. Sun, "Convergence and stability in collocation methods of equation," Journal of Applied Mathematics, vol. 2012, Article ID 125926, 17 pages, 2012.

[2] H. Shao and Q. L. Han, "New stability criteria for linear discrete-time systems with interval-like time-varying delays," IEEE Transactions on Automatic Control, vol. 56, no. 3, pp. 619$625,2011$.

[3] C. Li, J. Shi, and J. Sun, "Stability of impulsive stochastic differential delay systems and its application to impulsive stochastic neural networks," Nonlinear Analysis. Theory, Methods \& Applications, vol. 74, no. 10, pp. 3099-3111, 2011.

[4] C. Li, J. Sun, and R. Sun, "Stability analysis of a class of stochastic differential delay equations with nonlinear impulsive effects," Journal of the Franklin Institute, vol. 347, no. 7, pp. 1186-1198, 2010.

[5] S. Pan, J. Sun, and S. Zhao, "Stabilization of discrete-time markovian jump linear systems via time-delayed and impulsive controllers," Automatica, vol. 44, no. 11, pp. 2954-2958, 2008. 
[6] J. Sun, Y. Zhang, and Q. Wu, "Less conservative conditions for asymptotic stability of implusive control systems," IEEE Transactions on Automatic Control, vol. 48, no. 5, pp. 829-831, 2003.

[7] Q. Zhang and T. Ma, "A nonlinear Schrödinger model for manyparticle quantum systems," Journal of Applied Mathematics, vol. 2012, Article ID 387823, 15 pages, 2012.

[8] J. Barreiro, M. Müller, P. Schindler et al., "An open-system quantum simulator with trapped ions," Nature, vol. 470, no. 7335, pp. 486-491, 2011.

[9] C.-D. Yang, "Stability and quantization of complex-valued nonlinear quantum systems," Chaos, Solitons and Fractals, vol. 42, no. 2, pp. 711-723, 2009.

[10] R. Ceylan, M. Ceylan, Y. Özbay, and S. Kara, "Fuzzy clustering complex-valued neural network to diagnose cirrhosis disease," Expert Systems With Applications, vol. 38, no. 8, pp. 9744-9751, 2011.

[11] C. R. Doering, J. D. Gibbon, D. D. Holm, and B. Nicolaenko, "Low-dimensional behaviour in the complex Ginzburg-Landau equation," Nonlinearity, vol. 1, no. 2, pp. 279-309, 1988.

[12] S. Orszag, "Accurate solution of the orr-sommerfeld stability equation," Journal of Fluid Mechanics, vol. 50, no. 4, pp. 689703, 1971.

[13] M. Ráb, "Geometrical approach to the study of the Riccati differential equation with complex-valued coefficients," Journal of Differential Equations, vol. 25, no. 1, pp. 108-114, 1977.

[14] A. Rauh, L. Hannibal, and N. B. Abraham, "Global stability properties of the complex Lorenz model," Physica D, vol. 99, no. 1, pp. 45-58, 1996.

[15] G. M. Mahmoud, S. A. Aly, and A. A. Farghaly, "On chaos synchronization of a complex two coupled dynamos system," Chaos, Solitons and Fractals, vol. 33, no. 1, pp. 178-187, 2007.

[16] T. Fang and J. Sun, "Controllability and observability of complex [r]-matrix time-varying impulsive systems," Advances in Difference Equations, vol. 2013, no. 1, p. 129, 2013.

[17] S. Zhao and J. Sun, "Controllability and observability for impulsive systems in complex fields," Nonlinear Analysis, vol. 11, no. 3, pp. 1513-1521, 2010.

[18] J. Kalas, "On the asymptotic behaviour of the equation $d z / d t=$ $f(t, z)$ with a complex-valued function $f$,' Rch Cucamonga, vol. 17, no. 1, pp. 11-22, 1981.

[19] T. Fang and J. Sun, "Existence and uniqueness of solutions to complex-valued nonlinear impulsive differential systems," Advances in Difference Equations, vol. 2012, no. 1, p. 115, 2012.

[20] V. Lakshmikantham and S. Leela, Differential and Integral Inequalities: Theory and Applications Ordinary differential equations, vol. 1, Academic Press, 1969.

[21] W. Hahn, Stability of Motion, vol. 138, Springer, 1967. 


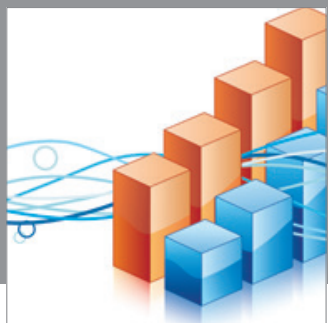

Advances in

Operations Research

mansans

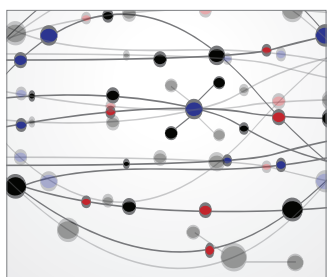

The Scientific World Journal
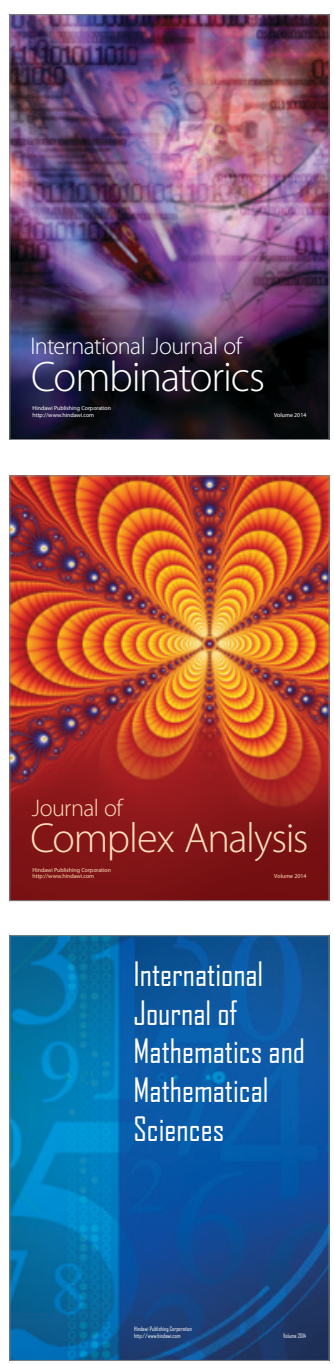
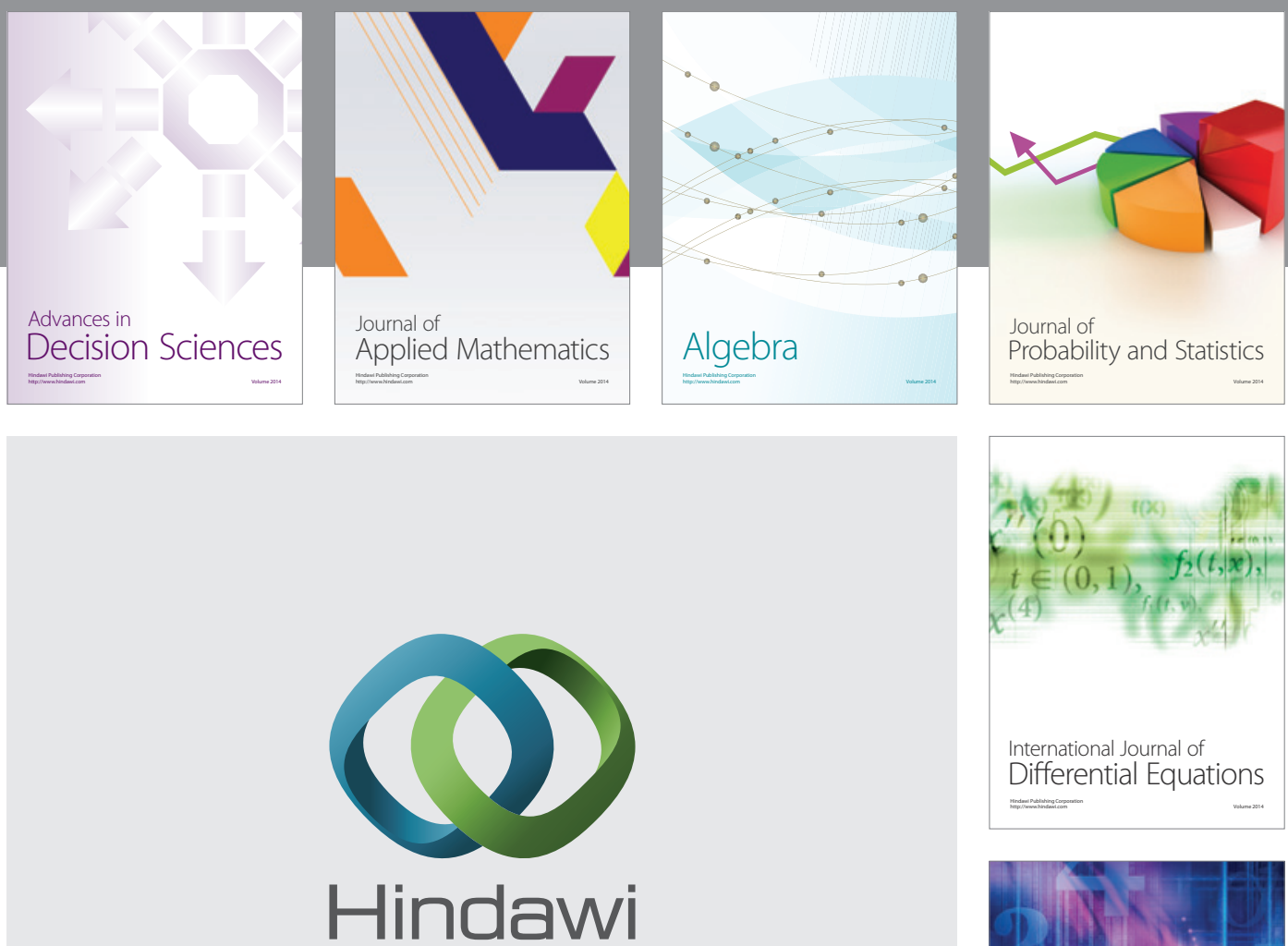

Submit your manuscripts at http://www.hindawi.com
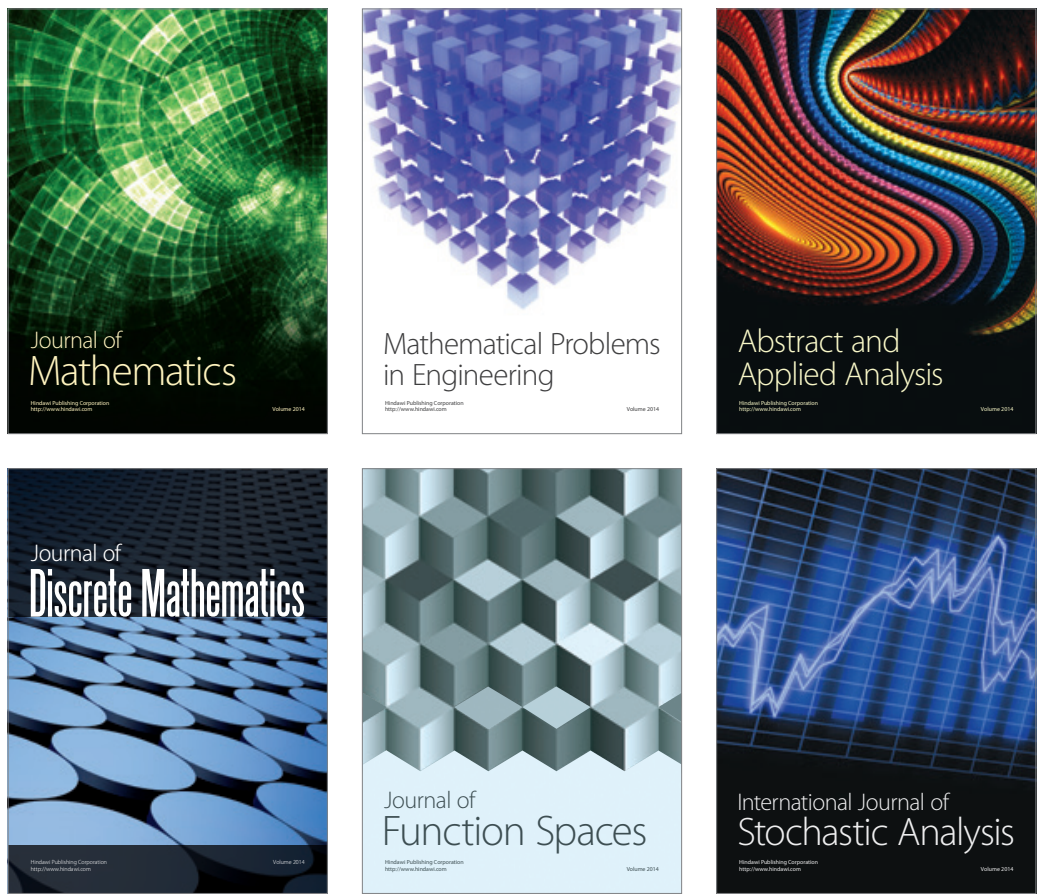

Journal of

Function Spaces

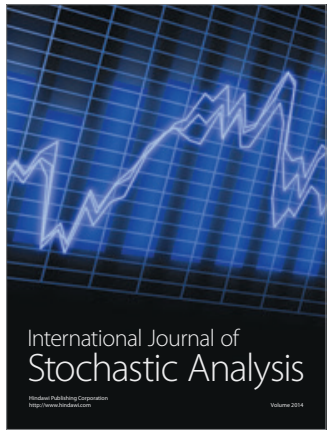

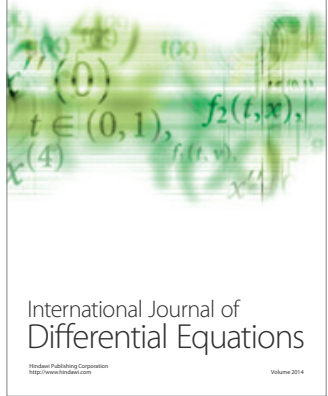
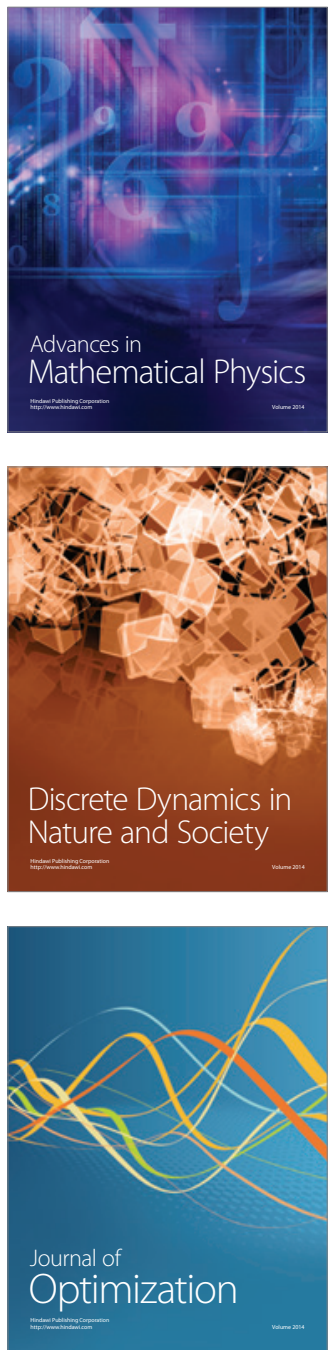